\title{
Comments on the cosmic convergence of nonexpansive maps
}

\author{
Armando W. Gutiérrez and Anders Karlsson
}

\begin{abstract}
This note discusses some aspects of the asymptotic behaviour of nonexpansive maps. Using metric functionals, we make a connection to the invariant subspace problem and prove a new result for nonexpansive maps of $\ell^{1}$. We also point out some inaccurate assertions appearing in the literature on this topic.
\end{abstract}

Mathematics Subject Classification. Primary 47H09; Secondary 47A15.

Keywords. Metric fixed point theory, nonexpansive maps, invariant subspace problem, metric functionals.

\section{Introduction}

This brief note was inspired by recent papers by Bauschke et al. [2], and Ryu $[26]$. Let $(X, d)$ be a metric space and let $T$ be a nonexpansive map of $X$ into itself, that is,

$$
d(T x, T y) \leq d(x, y)
$$

for all $x, y \in X$. The question concerns the asymptotic behaviour of the iterates $T^{n} x$ as $n \rightarrow \infty$. The first important case of this is well known: the contraction mapping principle. In this paper we are mostly interested in the complementary case; when no fixed point exists. The case when $X$ is a Banach space has been especially studied, see $[1,2,13,16-18,20,22,23,26]$ and references therein. It should be pointed out that a significant special case was considered in the 1930s: the mean ergodic theorem of von Neumann and Carleman, especially in the generality of F. Riesz [25, Theorem 1]. Here $X$ is a Hilbert space and

$$
T x=U x+v
$$

where $U$ is a linear operator of norm at most one and $v \in X$. Then the iterates $T^{n} 0$ converge in the following sense: 


$$
\lim _{n \rightarrow \infty} \frac{1}{n} T^{n} 0=\lim _{n \rightarrow \infty} \frac{1}{n} \sum_{k=0}^{n-1} U^{k} v=\pi_{U}(v),
$$

where $\pi_{U}$ is the projection onto the subspace of $U$-invariant vectors.

It is stated in [2, Fact 1.1] and also in [26] that for fixed-point free nonexpansive maps of Hilbert spaces the orbit must diverge in the sense that $\left\|T^{n} x\right\| \rightarrow \infty$ as $n \rightarrow \infty$. This may, however, fail in infinite-dimensional Hilbert spaces as an isometry discovered by Edelstein in the 1960s demonstrates, recalled in $[9$, p. 1453$]$ and the nice exposition $[27$, p. 6$]$. That is, in spaces which are not locally compact it may happen that the orbit $\left(T^{n} x\right)_{n \geq 0}$ neither stays bounded nor leaves every bounded set. On the other hand, this phenomenon cannot happen in spaces where closed bounded sets are compact as it was shown by Calka in the 1980s (a simple proof is given in [6, Lemma $2.6])$.

The quantity

$$
\tau=\lim _{n \rightarrow \infty} \frac{1}{n}\left\|T^{n} x\right\|
$$

always exists by a well-known subadditive argument and it is independent of $x$. Convergence in direction, cosmic convergence in the terminology of $[2,26]$, in the case $\tau>0$ for general Banach spaces was treated in $[13,20]$ among other papers. A very general result in this direction is found in [7] which considers random products of nonexpansive maps of any metric space. In the case $\tau=0$ one may wonder, as the authors in [2] do, if cosmic convergence still takes place, that is, whether

$$
\frac{T^{n} x}{\left\|T^{n} x\right\|}
$$

converges as $n \rightarrow \infty$. Strong and weak limits of this expression are called strong and weak cosmic limits respectively. This question is also raised in [10, Problem 4.6]. The paper [2] proves several interesting cases when this is true, and suggests a conjecture that it might always be true for finite dimensional Hilbert spaces. A counterexample was given in [26, Section 3]. In 2005, Enrico Le Donne showed the second author another such an example (unpublished). In other normed spaces, a counterexample was considered by Kohlberg and Neyman in [13, p. 272], also discussed in [6, p. 1936].

It still makes sense to wonder about what the limit set can be when it is not just one point. Theorem [11, Theorem 11] shows that it must be contained in certain kinds of sectors. This can be compared with Corollary 5.3 to Theorem 5.1 in [26]. These are some of the most general results presently available on this type of convergence known to us. When $X$ is hyperbolic there are many results starting from Wolff-Denjoy showing that cosmic convergence holds, for example [9], see also the discussion in [2]. Let us point out the similar question and conjecture in the case $X$ is a convex set equipped with Hilbert's metric, called the Karlsson-Nussbaum conjecture, see [14] for one of the most recent significant contributions.

In Sect. 3, we will prove the following connection of cosmic convergence to the problem of the existence of non-trivial closed invariant subspaces: 
Theorem. Suppose that $T$ is a nonexpansive map of a real Hilbert space into itself of the form $T x=U x+v$ for some vector $v$ and linear operator $U$ of norm at most one. If $0 \notin \overline{\operatorname{Im}(I-T)}$, then there is a non-zero continuous linear functional $f$ such that

$$
f(T x) \geq f(x)
$$

for all $x$. This inequality implies that $\operatorname{ker}(f)$ is a non-trivial closed invariant subspace for $U$.

In Sect. 4, we will prove:

Theorem. Let $T$ be a nonexpansive map of $\ell^{1}(\mathbb{Z})$ into itself. Then there is a non-trivial metric functional $h$ so that

$$
h(T x) \leq h(x)
$$

for every $x \in \ell^{1}(\mathbb{Z})$, and the orbit $\left(T^{n} 0\right)_{n \geq 0}$ is contained in a half-space. More precisely, there is a non-trivial continuous linear functional $f$ so that

$$
f\left(T^{n} 0\right) \geq 0
$$

for all $n \geq 0$.

Metric functionals, which constitute a main tool in this note will be recalled below. We provide some further statements and examples, such as Proposition 3 about firmly nonexpansive maps. We end by giving a simple example of a nonexpansive map whose unbounded orbits converge to a metric functional but without having any weak cosmic limit point. This shows once more that metric functionals are useful when linear notions fail to describe a phenomenon.

\section{Metric functionals}

Let $(X, d)$ be any metric space, for example a Banach space. Fix $x_{0} \in X$, in the case of a Banach space we take $x_{0}=0$. We consider the following map:

$$
\Phi: X \rightarrow \mathbb{R}^{X}
$$

via

$$
x \mapsto h_{x}(\cdot):=d(\cdot, x)-d\left(x_{0}, x\right) .
$$

The map $\Phi$ is injective (if $h_{x}=h_{y}$, then in particular $h_{x}(x)=h_{y}(x)$ and $h_{x}(y)=h_{y}(y)$ which together imply that $\left.d(x, y)=0\right)$. As the notation indicates, we endow the target space $\mathbb{R}^{X}$ with the topology of pointwise convergence. The map $\Phi$ is clearly continuous. The closure $\overline{\Phi(X)}$ is compact and consists of a subset of nonexpansive maps $h: X \rightarrow \mathbb{R}$ with $h\left(x_{0}\right)=0$. Every element of $\overline{\Phi(X)}$ is called a metric functional on $X$. See $[5,7,8,12]$ for discussions on metric functionals and the related notion of horofunctions.

Below we will use two cases:

Proposition $1[8,12]$. Let $H$ be a real Hilbert space with scalar product $(\cdot, \cdot)$. Every metric functional on $H$ has precisely one of the following forms: 
1. $h(x)=\|x\| ;$

2. $h(x)=\sqrt{\|x\|^{2}-2(x, r v)+r^{2}}-r$, with $0<r<\infty$ and $v \in H,\|v\|<1$;

3. $h(x)=\|x-r v\|-r$, with $0<r<\infty$ and $v \in H,\|v\|=1$;

4. $h(x)=-(x, v)$, with $v \in H,\|v\| \leq 1$.

Note that only the metric functionals of the form 4. are unbounded from below (except $h \equiv 0)$.

The first author determined all the metric functionals on $\ell^{1}(\mathbb{Z})$ :

Proposition 2 [8]. The following functions are precisely the metric functionals on $\ell^{1}(\mathbb{Z})$ :

$$
h(x)=\sum_{s \in I} \epsilon_{s} x_{s}+\sum_{s \in \mathbb{Z} \backslash I}\left|x_{s}-z_{s}\right|-\left|z_{s}\right|,
$$

where $I \subseteq \mathbb{Z}, \epsilon_{s} \in\{-1,+1\}$ for all $s \in I$ and $z_{s}$ are arbitrary real numbers for all $s \in \mathbb{Z} \backslash I$.

Another fact we will use below (see [7, Lemma 3.1]) is that for any metric functional $h$ on a Banach space there is always a continuous linear functional $f$ of norm at most 1 such that $f \leq h$. As a first illustration of this tool let us point out the following. Browder and Bruck introduced firmly nonexpansive maps of Banach spaces. Those are maps $T: V \rightarrow V$ of a Banach space $V$ (or a convex subsets thereof) satisfying

$$
\|T x-T y\| \leq\|(1-t)(T x-T y)+t(x-y)\|
$$

for every $x, y \in V$ and $t \geq 0$. Reich and Shafrir proved in [24] that for such maps it holds that

$$
\lim _{n \rightarrow \infty}\left\|T^{n+1} x-T^{n} x\right\|=\tau
$$

where $\tau$ is defined above in (1.1). This is a property used in the proof of [26, Theorem 5.1]. The following reproves the main theorem in [5, Section 3] in a very special case, but with the additional information that our metric functional $h$ is a limit point of the orbit $\left(T^{n} 0\right)_{n \geq 0}$ in the compact space $\overline{\Phi(V)}$.

Proposition 3. Let $T$ be a firmly nonexpansive map of a Banach space $V$ into itself with $\tau=0$. Then there is a metric functional $h$ on $V$ which is a limit point of $\left(T^{n} 0\right)_{n \geq 0}$ in the compact space $\overline{\Phi(V)}$ and such that

$$
h(T x) \leq h(x)
$$

for all $x \in V$.

Proof. Take a subsequence $n_{i}$ so that $h_{T^{n_{i} 0}} \rightarrow h$ (in the case that $\overline{\Phi(V)}$ is not sequentially compact we can use a similar argument as in [7, p. 1907]). Note that for every $x \in V$, 


$$
\begin{aligned}
h(T x) & =\lim _{i \rightarrow \infty}\left\|T x-T^{n_{i}} 0\right\|-\left\|T^{n_{i}} 0\right\| \\
& \leq \liminf _{i \rightarrow \infty}\left\|x-T^{n_{i}-1} 0\right\|-\left\|T^{n_{i}} 0\right\| \\
& \leq \liminf _{i \rightarrow \infty}\left\|x-T^{n_{i}} 0\right\|+\left\|T^{n_{i}} 0-T^{n_{i}-1} 0\right\|-\left\|T^{n_{i}} 0\right\| \\
& =h(x)+\tau .
\end{aligned}
$$

If $\tau=0$, then the proposition is proved.

Note that we do not need $T$ to be defined on the whole vector space, just as long as we can iterate the map. Firmly nonexpansive maps are of interest in the study of certain non-linear operators as pioneered by Browder and they often arise in optimization problems; see, for example, [3,4,21].

Ryu constructed an interesting firmly nonexpansive map $T$ of $\ell^{2}(\mathbb{N})$ into itself $\left[26\right.$, p. 11] such that $T^{n} 0 /\left\|T^{n} 0\right\|$ converges weakly, but not strongly, to 0 . We note that the iterates $T^{n} 0$ converge to the metric functional $h=0$. Furthermore we note that Ryu's map also provides a nonexpansive map of $\ell^{1}(\mathbb{N})$ into itself, and that in this case the iterates $T^{n} 0$ converge to a nontrivial metric functional (which in fact is linear). Indeed, the result in [26, Lemma 4.1] implies that for fixed $i$, the $i$ th coefficient of the $k$ th iterate looks like $\left(T^{k} 0\right)_{i}=\log k+O(1)$ as $k \rightarrow \infty$. Now if we consider $x \in \ell^{1}$ for which only a finite number of coefficients are non-zero, say $F$ is the support of $x$, then

$$
\left\|x-T^{k} 0\right\|-\left\|T^{k} 0\right\|=\sum_{i \in F}\left|x_{i}-\left(T^{k} 0\right)_{i}\right|-\left|\left(T^{k} 0\right)_{i}\right|
$$

which converges to $-\sum_{i \in F} x_{i}$ as $k \rightarrow \infty$. Since such finitely supported points are dense in $\ell^{1}$ we have that the iterates $T^{k} 0$ converge to the metric functional, indeed linear, $h(x)=-\sum_{i \geq 0} x_{i}$.

\section{Invariant subspaces}

One of the oldest and best-known open problems in operator theory is the invariant subspace problem. It asks whether every bounded linear operator of a complex Hilbert space $H$ of dimension at least two must have a nontrivial invariant closed linear subspace. For general Banach spaces the first counterexample was found by Enflo and other examples by Read [19]. We will here make a connection to the topic of the present paper.

First we point out that in Remark 3.1 of [13] it is stated that one can choose a linear functional $f$ of norm 1 such that $f\left(T^{n} x-x\right) \geq n \tau$. But it seems to us that this can only be guaranteed when $\tau>0$, otherwise the weak limit of linear functionals in the proof may be $f \equiv 0$. Compare also with [5, p. 355].

Given any bounded linear operator $U: H \rightarrow H$, by rescaling we can assume that its norm is at most one since this does not alter the invariant subspaces. We will consider associated affine nonexpansive maps, that is, $T x=U x+v$ for some vector $v \in H$. We will be interested in statements of the form that there exists a linear functional $f$ such that 


$$
f(T x) \geq f(x)
$$

for all $x \in H$.

Theorem 1. Suppose that $T$ is a nonexpansive map of a real Hilbert space into itself of the form $T x=U x+v$ for some vector $v$ and linear operator $U$ of norm at most one. If $0 \notin \overline{\operatorname{Im}(I-T)}$, then there is a non-zero continuous linear functional $f$ such that

$$
f(T x) \geq f(x)
$$

for all $x$. This inequality implies that $\operatorname{ker}(f)$ is a non-trivial closed invariant subspace for $U$.

Proof. It is known since Pazy's work that the condition imposed implies that the corresponding escape rate $\tau$ defined above in (1.1) is strictly positive. This also follows from the main theorem in Gaubert-Vigeral [5] that, moreover, asserts that there is a metric functional such that

$$
h(T x) \leq h(x)-\tau
$$

for every $x$ in the Hilbert space. Since $\tau>0$, it follows from the above inequality iterated that $h\left(T^{n} x\right) \rightarrow-\infty$ as $n \rightarrow \infty$. This shows, in view of the identification of the metric functionals in a Hilbert space recalled in Proposition 1, that $h$ must be of the type $h(x)=-(x, q)$ for some nonzero vector $q$ which must have norm 1 . Hence, $f(x):=-h(x)$ is the linear functional as required.

Notice now that this means that $-(U x+v, q) \leq-(x, q)$ for every $x$. In particular, for any $x \in \operatorname{ker} f$, we have $(U x, q)+(v, q) \geq 0$. Applying the same inequality with $t x$ with scalars $t \in \mathbb{R}$ instead of $x$, using linearity of the scalar product, it follows that the only possibility is that $(U x, q)=0$, in other words $U x \in \operatorname{ker} f$. Hence, the kernel of $f$, which clearly is a non-trivial and closed linear subspace is invariant under $U$.

There is a hope, related to cosmic convergence, that even when $0 \in$ $\overline{\operatorname{Im}(I-T)} \backslash \operatorname{Im}(I-T)$, there is sometimes such a linear functional $f$. (The case $0 \in \operatorname{Im}(I-T)$ means that $v=x-U x$ for a certain $x)$. It is stated in [26, Theorem 5.1] that for a non-zero cosmic weak limit point $q$, it holds that $(T x-x, q) \geq 0$ for all $x$ in the Hilbert space, which is precisely what is needed in view of Theorem 1. Note, however, that in the proof of [26, Theorem 5.1], it is assumed that $\left\|T^{k_{j}+1} x-T^{k_{j}} x\right\| \rightarrow 0$, but for an isometry this norm is constant and positive unless $x$ is a fixed point. As far as we can see, the proof of [26, Theorem 5.1] needs this assumption.

Remark 1. The condition in Theorem 1 means that 1 is in the compression spectrum of the linear operator $U$. By the definition of this subset of the spectrum, the set $\overline{I m(I-U)}$ is a non-trivial closed subspace which is clearly invariant under $U$. In contrast, Theorem 1 provides a co-dimension 1 closed invariant subspace for $U$. 


\section{Nonexpansive maps of $\ell^{1}$}

In view of Theorem 1 above, it is interesting to recall that for any nonexpansive map $T$ of a Banach space, Gaubert and Vigeral in their paper [5] (strengthening [9] in the case of star-shaped hemi-metrics) provide a metric functional $h$ so that

$$
h(T x) \leq h(x)
$$

for every $x$. For a Hilbert space, in case $\tau=0$ there seems to be no way in general of guaranteeing that $h$ is not the function identically 0 . On the other hand, Gutiérrez [8] identified explicitly all the metric functionals of the Banach spaces $\ell^{1}$ recalled in Proposition 2, and none is identically 0 . So this gives finer information than [13] in this case. It is pointed out in [7, Lemma 3.1] that there is always a continuous linear functional $f$ of norm at most 1 such that $f \leq h$. Nevertheless, the discrepancy between linear and metric functionals has as consequence that the proof of Theorem 1 does not lead to an affirmative solution to the invariant subspace problem in the case of $\ell^{1}$. Indeed, there is a celebrated counterexample to the invariant subspace problem for $\ell^{1}$, constructed by Read [19].

The following can be said about nonexpansive maps of $\ell^{1}$ :

Theorem 2. Let $T$ be a nonexpansive map of $\ell^{1}(\mathbb{Z})$ into itself. Then there is a non-trivial metric functional $h$ so that

$$
h(T x) \leq h(x)
$$

for every $x \in \ell^{1}(\mathbb{Z})$, and the orbit $\left(T^{n} 0\right)_{n \geq 0}$ is contained in a half-space. More precisely, there is a non-trivial continuous linear functional $f$ so that

$$
f\left(T^{n} 0\right) \geq 0
$$

for all $n \geq 0$.

Proof. The first statement is the Gaubert-Vigeral Theorem [5, Theorem 1] with the addition of the explicit determination of such metric functionals in [8], see Proposition 2 above. Each of these metric functionals takes on negative values somewhere, except for $h(x)=\|x\|$. In this latter exceptional case, note that $h(T x) \leq h(x)$ means that $\|T x\| \leq\|x\|$ which applied to $x=0$ gives $T(0)=0$. In this case, any linear functional $f$ would satisfy our claim since $f\left(T^{n} 0\right)=f(0)=0$. If $h$ does take negative values then take the linear functional $g$ obtained in [7, Lemma 3.1] with $g \leq h$. This linear functional must be non-trivial since it is forced to take on strictly negative values at some points. Iterating the main inequality, we have

$$
h\left(T^{n} 0\right) \leq h\left(T^{n-1} 0\right) \leq \cdots \leq h(0)=0 .
$$

Let finally $f(x)=-g(x)$, of course also not the identically 0 linear functional, and with the property $f\left(T^{n} 0\right) \geq-h\left(T^{n} 0\right) \geq 0$.

Let us remark that Edelstein's example alluded to already above extends to $\ell^{1}(\mathbb{Z})$. This isometry has unbounded orbits but they nevertheless return infinitely often to a fixed bounded set. Let us finish by another example: 
Example 1. Consider the nonexpansive map $T: \ell^{1}(\mathbb{N}) \rightarrow \ell^{1}(\mathbb{N})$ defined by $T\left(x_{1}, x_{2}, \ldots\right)=\left(1, x_{1}, x_{2}, \ldots\right)$. This map clearly has no fixed points in $\ell^{1}(\mathbb{N})$. Indeed the orbits tend to infinity, more precisely, $T^{n} 0=(1,1,1, \ldots, 1,0,0, \ldots)$ and $h_{T^{n} 0}$ converges to

$$
h(x)=\sum_{s=1}^{\infty}\left|x_{s}-1\right|-1
$$

as $n \rightarrow \infty$. This $h$ is the metric functional that is obtained in $[9,12]$ (also the one in [5] as can be verified), and clearly $h\left(T^{n} 0\right) \rightarrow-\infty$. On the other hand, there are no weak cosmic limit points.

\section{Acknowledgements}

The first author acknowledges financial support from the Vilho, Yrjö and Kalle Väisälä Foundation of the Finnish Academy of Science and Letters. The second author acknowledges partial financial support from the Swiss NSF grant 200020_15958.

Funding Open Access funding provided by Université de Genève.

Open Access. This article is licensed under a Creative Commons Attribution 4.0 International License, which permits use, sharing, adaptation, distribution and reproduction in any medium or format, as long as you give appropriate credit to the original author(s) and the source, provide a link to the Creative Commons licence, and indicate if changes were made. The images or other third party material in this article are included in the article's Creative Commons licence, unless indicated otherwise in a credit line to the material. If material is not included in the article's Creative Commons licence and your intended use is not permitted by statutory regulation or exceeds the permitted use, you will need to obtain permission directly from the copyright holder. To view a copy of this licence, visit http:// creativecommons.org/licenses/by/4.0/.

Publisher's Note Springer Nature remains neutral with regard to jurisdictional claims in published maps and institutional affiliations.

\section{References}

[1] Baillon, J.B., Bruck, R.E., Reich, S.: On the asymptotic behavior of nonexpansive mappings and semigroups in Banach spaces. Houston J. Math. 4, 1-9 (1978)

[2] Bauschke, H.H., Douglas, G.R., Moursi, W.M.: On a result of Pazy concerning the asymptotic behaviour of non-expansive mappings. J. Fixed Point Theory Appl. 18(2), 297-307 (2016)

[3] Bauschke, H.H., Matoušková, E., Reich, S.: Projection and proximal point methods: convergence results and counterexamples. Nonlinear Anal. 56(5), $715-738$ (2004)

[4] Bruck, R.E., Reich, S.: Nonexpansive projections and resolvents of accretive operators in Banach spaces. Houston J. Math. 3(4), 459-470 (1977) 
[5] Gaubert, S., Vigeral, G.: A maximin characterisation of the escape rate of nonexpansive mappings in metrically convex spaces. Math. Proc. Camb. Philos. Soc. 152(2), 341-363 (2012)

[6] Gouëzel, S.: Subadditive cocycles and horofunctions. Proceedings of the International Congress of Mathematicians-Rio de Janeiro 2018. Vol. III. Invited lectures, 1933-1947, World Sci. Publ., Hackensack, NJ (2018)

[7] Gouëzel, S., Karlsson, A.: Subadditive and multiplicative ergodic theorems. J. Eur. Math. Soc. (JEMS) 22(6), 1893-1915 (2020)

[8] Gutiérrez, A.W.: On the metric compactification of infinite-dimensional $\ell_{p}$ spaces. Can. Math. Bull. 62(3), 491-507 (2019)

[9] Karlsson, A.: Non-expanding maps and Busemann functions. Ergodic Theory Dyn. Syst. 21(5), 1447-1457 (2001)

[10] Karlsson, A.: Nonexpanding maps, Busemann functions, and multiplicative ergodic theory. Rigidity in dynamics and geometry (Springer, Berlin, pp. 283294, 2002). (2000)

[11] Karlsson, A.: On the dynamics of isometries. Geom. Topol. 9, 2359-2394 (2005)

[12] Karlsson, A.: Elements of a metric spectral theory. arXiv:1904.01398

[13] Kohlberg, E., Neyman, A.: Asymptotic behavior of nonexpansive mappings in normed linear spaces. Israel J. Math. 38(4), 269-275 (1981)

[14] Lemmens, B., Lins, B., Nussbaum, R., Wortel, M.: Denjoy-Wolff theorems for Hilbert's and Thompson's metric spaces. J. Anal. Math. 134(2), 671-718 (2018)

[15] Lins, B.: Asymptotic behavior of nonexpansive mappings in finite dimensional normed spaces. Proc. Am. Math. Soc. 137(7), 2387-2392 (2009)

[16] Matoušková, E., Reich, S.: Reflexivity and approximate fixed points. Stud. Math. 159(3), 403-415 (2003)

[17] Pazy, A.: Asymptotic behavior of contractions in Hilbert space. Israel J. Math. 9, 235-240 (1971)

[18] Plant, A.T., Reich, S.: The asymptotics of nonexpansive iterations. J. Funct. Anal. 54(3), 308-319 (1983)

[19] Read, C.J.: A solution to the invariant subspace problem on the space $l^{1}$. Bull. Lond. Math. Soc. 17(4), 305-317 (1985)

[20] Reich, S.: Asymptotic behavior of contractions in Banach spaces. J. Math. Anal. Appl. 44, 57-70 (1973)

[21] Reich, S.: Extension problems for accretive sets in Banach spaces. J. Funct. Anal. 26(4), 378-395 (1977)

[22] Reich, S.: On the asymptotic behavior of nonlinear semigroups and the range of accretive operators. J. Math. Anal. Appl. 79, 113-126 (1981)

[23] Reich, S.: On the asymptotic behavior of nonlinear semigroups and the range of accretive operators. II. J. Math. Anal. Appl. 87, 134-146 (1982)

[24] Reich, S., Shafrir, I.: The asymptotic behavior of firmly nonexpansive mappings. Proc. Am. Math. Soc. 101(2), 246-250 (1987)

[25] Riesz, F.: Some mean ergodic theorems. J. Lond. Math. Soc. 13(4), 274-278 (1938)

[26] Ryu, E.K.: Cosmic divergence, weak cosmic convergence, and fixed points at infinity. J. Fixed Point Theory Appl. 20(3), 109 (2018) 
[27] Valette, A.: Affine isometric actions on Hilbert spaces and amenability. Lectures at ESI, Vienna in 2007. https://www.unine.ch/files/live/sites/math/ files/shared/documents/articles/ESIValette.pdf

Armando W. Gutiérrez

INRIA Saclay and CMAP Ecole Polytechnique

CNRS

91128 Palaiseau

France

e-mail: armando.w.gutierrez@inria.fr;

armando.gutierrez@polytechnique.edu

Anders Karlsson

Section de Mathématiques

Université de Genève

2-4 Rue du Lièvre, Case Postale 64

1211 Genève 4

Switzerland

e-mail: anders.karlsson@unige.ch;

anders.karlsson@math.uu.se

and

Matematiska Institutionen

Uppsala Universitet

Box 25675105 Uppsala

Sweden

Accepted: August 23, 2021. 